\title{
ON THE QUASI-CONFORMAL CURVATURE TENSOR OF AN ALMOST KENMOTSU MANIFOLD WITH NULLITY DISTRIBUTIONS
}

\author{
Dibakar Dey and Pradip Majhi
}

\begin{abstract}
The objective of the present paper is to characterize quasi-conformally flat and $\xi$-quasi-conformally flat almost Kenmotsu manifolds with $(k, \mu)$-nullity and $(k, \mu)^{\prime}$-nullity distributions, respectively. Also we characterize almost Kenmotsu manifolds with vanishing extended quasi-conformal curvature tensor and extended $\xi$-quasiconformally flat almost Kenmotsu manifolds such that the characteristic vector field $\xi$ belongs to the $(k, \mu)$-nullity distribution.

Keywords: Almost Kenmotsu manifold, Einstein manifold, Weyl conformal curvature tensor, Quasi-conformal curvature tensor, Extended quasi-conformal curvature tensor.
\end{abstract}

\section{Introduction}

Let $M$ be a $(2 n+1)$-dimensional Riemannian manifold with metric $g$ and let $T(M)$ be the Lie algebra of differentiable vector fields in $M$. The Ricci operator $Q$ of $(M, g)$ is defined by

$$
g(Q X, Y)=S(X, Y),
$$

where $S$ denotes the Ricci tensor of type $(0,2)$ on $M$ and $X, Y \in T(M)$. The Weyl conformal curvature tensor $C$ is defined by

$$
\begin{aligned}
C(X, Y) Z= & R(X, Y) Z-\frac{1}{2 n-1}[S(Y, Z) X-S(X, Z) Y+g(Y, Z) Q X \\
& -g(X, Z) Q Y]+\frac{r}{2 n(2 n-1)}[g(Y, Z) X-g(X, Z) Y],
\end{aligned}
$$

for $X, Y, Z \in T(M)$, where $R$ and $r$ denote the Riemannian curvature tensor and scalar curvature of $M$, respectively.

Received February 08, 2018; accepted May 05, 2018

2010 Mathematics Subject Classification. Primary 53C25; Secondary 53C35 
For a $(2 n+1)$-dimensional Riemannian manifold, the quasi-conformal curvature tensor $\tilde{C}$ is given by

$\tilde{C}(X, Y) Z=a R(X, Y) Z+b[S(Y, Z) X-S(X, Z) Y+g(Y, Z) Q X-g(X, Z) Q Y]$

$$
-\frac{r}{2 n+1}\left[\frac{a}{2 n}+2 b\right][g(Y, Z) X-g(X, Z) Y]
$$

where $a$ and $b$ are two scalars. The notion of quasi-conformal curvature tensor was introduced by Yano and Sawaki [21]. If $a=1$ and $b=-\frac{1}{2 n-1}$, then the quasiconformal curvature tensor reduces to conformal curvature tensor.

A $(2 n+1)$-dimensional Riemannian manifold will be called a manifold of the quasi-constant curvature if the Riemannian curvature tensor $\tilde{R}$ of type $(0,4)$ satisfies the condition

$$
\begin{aligned}
\tilde{R}(X, Y, Z, W)= & p[g(Y, Z) g(X, W)-g(X, Z) g(Y, W)] \\
& +q[g(X, W) T(Y) T(Z)-g(X, Z) T(Y) T(W) \\
& +g(Y, Z) T(X) T(W)-g(Y, W) T(X) T(Z)],
\end{aligned}
$$

where $\tilde{R}(X, Y, Z, W)=g(R(X, Y) Z, W), p, q$ are scalars and there exists a unit vector field $\rho$ satisfying $g(X, \rho)=T(X)$. The notion of the quasi-constant curvature for Riemannian manfiolds was introduced by Chen and Yano [4].

At present, the study of nullity distributions is a very interesting topic on almost contact metric manifolds. The notion of $k$-nullity distribution was introduced by Gray [10] and Tanno [15] in the study of Riemannian manifolds $(M, g)$, which is defined for any $p \in M$ and $k \in \mathbb{R}$ as follows:

$$
N_{p}(k)=\left\{Z \in T_{p} M: R(X, Y) Z=k[g(Y, Z) X-g(X, Z) Y]\right\},
$$

for any $X, Y \in T_{p} M$, where $T_{p} M$ denotes the tangent vector space of $M$ at any point $p \in M$ and $R$ denotes the Riemannian curvature tensor of type $(1,3)$. Blair, Koufogiorgos and Papantonio [1] introduced the generalized notion of $k$-nullity distribution, named $(k, \mu)$-nullity distribution on a contact metric manifold $\left(M^{2 n+1}\right.$, $\phi, \xi, \eta, g)$, which is defined for any $p \in M$ and $k, \mu \in \mathbb{R}$ as follows:

$$
\begin{aligned}
N_{p}(k, \mu)=\left\{Z \in T_{p} M: R(X, Y) Z=\right. & k[g(Y, Z) X-g(X, Z) Y] \\
& +\mu[g(Y, Z) h X-g(X, Z) h Y]\},
\end{aligned}
$$

where $h=\frac{1}{2} £_{\xi} \phi$ and $£$ denotes the Lie differentiation.

In [7] Dileo and Pastore introduce the notion of $(k, \mu)^{\prime}$-nullity distribution, another generalized notion of $k$-nullity distribution, on an almost Kenmotsu manifold $\left(M^{2 n+1}, \phi, \xi, \eta, g\right)$, which is defined for any $p \in M^{2 n+1}$ and $k, \mu \in \mathbb{R}$ as follows:

$$
\begin{aligned}
N_{p}(k, \mu)^{\prime}=\left\{Z \in T_{p} M: R(X, Y) Z=\right. & k[g(Y, Z) X-g(X, Z) Y] \\
& \left.+\mu\left[g(Y, Z) h^{\prime} X-g(X, Z) h^{\prime} Y\right]\right\},
\end{aligned}
$$


where $h^{\prime}=h \circ \phi$.

A differentiable $(2 n+1)$-dimensional manifold $M$ is said to have a $(\phi, \xi, \eta)$ structure or an almost contact structure, if it admits a $(1,1)$ tensor field $\phi$, a characteristic vector field $\xi$ and a 1-form $\eta$ satisfying ([2],[3]),

$$
\phi^{2}=-I+\eta \otimes \xi, \eta(\xi)=1,
$$

where $I$ denotes the identity endomorphism. Here also $\phi \xi=0$ and $\eta \circ \phi=0$ hold; both can be derived from (1.8) easily.

If a manifold $M$ with a $(\phi, \xi, \eta)$-structure admits a Riemannian metric $g$ such that

$$
g(\phi X, \phi Y)=g(X, Y)-\eta(X) \eta(Y),
$$

for any vector fields $X, Y$ of $T_{p} M^{2 n+1}$, then $M$ is said to be an almost contact metric manifold. The fundamental 2 -form $\Phi$ on an almost contact metric manifold is defined by $\Phi(X, Y)=g(X, \Phi Y)$ for any $X, Y$ of $T_{p} M^{2 n+1}$. The condition for an almost contact metric manifold being normal is equivalent to the vanishing of the $(1,2)$-type torsion tensor $N_{\phi}$, defined by $N_{\phi}=[\phi, \phi]+2 d \eta \otimes \xi$, where $[\phi, \phi]$ is the Nijenhuis torsion of $\phi[2]$. Recently in ([7],[8],[9],[13],[14]), an almost contact metric manifold such that $\eta$ is closed and $d \Phi=2 \eta \wedge \Phi$ are studied and called almost Kenmotsu manifolds. Obviously, a normal almost Kenmotsu manifold is a Kenmotsu manifold. Also, Kenmotsu manifolds can be characterized by $\left(\nabla_{X} \phi\right) Y=g(\phi X, Y) \xi-\eta(Y) \phi X$, for any vector fields $X, Y$. It is well known [11] that a Kenmotsu manifold $M^{2 n+1}$ is locally a warped product $I \times_{f} N^{2 n}$ where $N^{2 n}$ is a Kähler manifold, $I$ is an open interval with coordinate $t$ and the warping function $f$, defined by $f=c e^{t}$ for some positive constant c. Let us denote the distribution orthogonal to $\xi$ by $\mathcal{D}$ and defined by $\mathcal{D}=\operatorname{Ker}(\eta)=\operatorname{Im}(\phi)$. In an almost Kenmotsu manifold, since $\eta$ is closed, $\mathcal{D}$ is an integrable distribution.

At each point $p \in M$, we have

$$
T_{p}(M)=\phi\left(T_{p}(M)\right) \oplus\left\{\xi_{p}\right\}
$$

where $\left\{\xi_{p}\right\}$ is 1-dimensional linear subspace of $T_{p}(M)$ generated by $\xi_{p}$. Then the Weyl conformal curvature tensor $C$ is a map:

$$
C: T_{p}(M) \times T_{p}(M) \times T_{p}(M) \rightarrow \phi\left(T_{p}(M)\right) \oplus\{\xi\} .
$$

Three particular cases can be considered as follows:

(1) $C: T_{p}(M) \times T_{p}(M) \times T_{p}(M) \rightarrow\{\xi\}$, that is, the projection of the image of $C$ in $\phi\left(T_{p}(M)\right)$ is zero .

(2) $C: T_{p}(M) \times T_{p}(M) \times T_{p}(M) \rightarrow \phi\left(T_{p}(M)\right)$, that is, the projection of the image of $C$ in $\{\xi\}$ is zero.

(3) $C: T_{p}(M) \times T_{p}(M) \times T_{p}(M) \rightarrow\{\xi\}$, that is, when $C$ is restricted to $\phi\left(T_{p}(M)\right) \times$ $\phi\left(T_{p}(M)\right)$, the projection of the image of $C$ in $\phi\left(T_{p}(M)\right)$ is zero, which is equivalent to $\phi^{2} C(\phi X, \phi Y) \phi Z=0$. 
Definition 1.1. [22] A contact metric manifold $\left(M^{2 n+1}, \phi, \xi, \eta, g\right)$ is said to be $\xi$-conformally flat if the linear operator $C(X, Y)$ is an endomorphism of $\phi(T(M))$, that is, if

$$
C(X, Y) \phi(T(M)) \subset \phi(T(M)) \text {. }
$$

Then it follows immediately that

Proposition 1.1. [22] On a contact metric manifold $\left(M^{2 n+1}, \phi, \xi, \eta, g\right)$, the following conditions are equivalent.

(a) $M^{2 n+1}$ is $\xi$-conformally flat,

(b) $\eta(C(X, Y) Z)=0$,

(c) $\phi^{2} C(X, Y) Z=-C(X, Y) Z$,

(d) $C(X, Y) \xi=0$,

where $X, Y, Z \in T(M)$.

Almost Kenmotsu manifolds have been studied by several authors such as Dileo and Pastore ([7]-[9]), Wang and Liu ([16]-[20]), De and Mandal([5], [6], [12]) and many others. In the present paper we like to study quasi-conformal curvature tensor of almost Kenmotsu manifolds with $(k, \mu)$ and $(k, \mu)^{\prime}$-nullity distributions, respectively. Also, we discuss vanishing extended quasi-conformal curvature tensor in an almost Kenmotsu manifold and extended $\xi$-quasi-conformally flat almost Kenmotsu manifolds with $(k, \mu)$-nullity distribution.

The paper is organized as follows:

In Section 2, we give a brief account on almost Kenmotsu manifolds with $\xi$ belonging to the $(k, \mu)$-nullity distribution and $\xi$ belonging to the $(k, \mu)^{\prime}$-nullity distribution. Section 3 deals with quasi-conformally flat and $\xi$-quasi-conformally flat almost Kenmotsu manifolds with the characteristic vector field $\xi$ belonging to the $(k, \mu)$-nullity distribution. As a consequence of the main result, we obtain several corollaries. Section 4 is devoted to the study of quasi-conformally flat almost Kenmotsu manifolds with the characteristic vector field $\xi$ belonging to the $(k, \mu)^{\prime}$-nullity distribution. In the final section, we discuss vanishing extended quasi-conformal curvature tensor in an almost Kenmotsu manifold and extended $\xi$-quasi-conformally flat almost Kenmotsu manifolds with $(k, \mu)$-nullity distribution.

\section{Almost Kenmotsu manifolds}

Let $M^{2 n+1}$ be an almost Kenmotsu manifold. We denote by $h=\frac{1}{2} £_{\xi} \phi$ and $l=$ $R(\cdot, \xi) \xi$ on $M^{2 n+1}$. The tensor fields $l$ and $h$ are symmetric operators and satisfy the following relations [13]:

$$
\begin{gathered}
h \xi=0, l \xi=0, \operatorname{tr}(h)=0, \operatorname{tr}(h \phi)=0, h \phi+\phi h=0, \\
\nabla_{X} \xi=X-\eta(X) \xi-\phi h X\left(\Rightarrow \nabla_{\xi} \xi=0\right),
\end{gathered}
$$




$$
\phi l \phi-l=2\left(h^{2}-\phi^{2}\right),
$$

$(2.4) R(X, Y) \xi=\eta(X)(Y-\phi h Y)-\eta(Y)(X-\phi h X)+\left(\nabla_{Y} \phi h\right) X-\left(\nabla_{X} \phi h\right) Y$,

for any vector fields $X, Y$. The $(1,1)$-type symmetric tensor field $h^{\prime}=h \circ \phi$ is anti-commuting with $\phi$ and $h^{\prime} \xi=0$. Also it is clear that ([7], [18])

$$
h=0 \Leftrightarrow h^{\prime}=0, \quad h^{\prime 2}=(k+1) \phi^{2}\left(\Leftrightarrow h^{2}=(k+1) \phi^{2}\right) .
$$

\section{Quasi-conformally flat almost Kenmotsu manifolds with $\xi$ belonging to the $(k, \mu)$-nullity distribution}

In this section we study quasi-conformally flat and $\xi$-quasi-conformally flat almost Kenmotsu manifolds with $\xi$ belonging to the $(k, \mu)$-nullity distribution.

From (1.6) we obtain

$$
R(X, Y) \xi=k[\eta(Y) X-\eta(X) Y]+\mu[\eta(Y) h X-\eta(X) h Y],
$$

where $k, \mu \in \mathbb{R}$. Before proving our main results in this section we first state the following:

Lemma 3.1. [7] Let $M^{2 n+1}$ be an almost Kenmotsu manifold of dimension $(2 n+$ $1)$. Suppose that the characteristic vector field $\xi$ belonging to the $(k, \mu)$-nullity distribution. Then $k=-1, h=0$ and $M^{2 n+1}$ is locally a wrapped product of an open interval and an almost Kähler manifold.

In view of Lemma 3.1 it follows from the equation (3.1),

$$
\begin{gathered}
R(X, Y) \xi=\eta(X) Y-\eta(Y) X, \\
R(\xi, X) Y=-g(X, Y) \xi+\eta(Y) X, \\
S(X, \xi)=-2 n \eta(X), \\
Q \xi=-2 n \xi,
\end{gathered}
$$

for any vector fields $X, Y$ on $M^{2 n+1}$.

Theorem 3.1. An almost Kenmotsu manifold $M^{2 n+1}$ with $\xi$ belonging to the $(k, \mu)$-nullity distribution is quasi-conformally flat if and only if the manifold is locally isometric to the hyperbolic space $\mathbb{H}^{2 n+1}(-1)$.

Proof: Let us first consider the manifold $M^{2 n+1}$ which is quasi-conformally flat, that is,

$$
\tilde{C}(X, Y) Z=0,
$$


for any vector fields $X, Y, Z$ on $M^{2 n+1}$.

From (1.3) we have

$$
\begin{aligned}
\tilde{R}(X, Y, Z, W)= & \frac{b}{a}[S(X, Z) g(Y, W)-S(Y, Z) g(X, W) \\
& +S(Y, W) g(X, Z)-S(X, W) g(Y, Z)] \\
& +\frac{r}{a(2 n+1)}\left[\frac{a}{2 n}+2 b\right][g(Y, Z) g(X, W)-g(X, Z) g(Y, W)] .
\end{aligned}
$$

Putting $Z=\xi$ in the above equation and using (3.2) and (3.4) we get

$$
\begin{aligned}
\eta(X) g(Y, W)-\eta(Y) g(X, W)= & \frac{b}{a}[-2 n \eta(X) g(Y, W)+2 n \eta(Y) g(X, W) \\
& +S(Y, W) \eta(X)-S(X, W) \eta(Y)] \\
& +\frac{r}{a(2 n+1)}\left[\frac{a}{2 n}+2 b\right][g(X, W) \eta(Y) \\
& -g(Y, W) \eta(X)] .
\end{aligned}
$$

Putting $Y=\xi$ in the above equation we obtain after simplification

$$
S(X, W)=\alpha g(X, W)+\beta \eta(X) \eta(W),
$$

where $\alpha=\frac{a}{b}\left[\frac{2 b n}{a}+\frac{r}{a(2 n+1)}\left[\frac{a}{2 n}+2 b\right]+1\right]$ and $\beta=\frac{a}{b}\left[-\frac{4 b n}{a}-\frac{r}{a(2 n+1)}\left[\frac{a}{2 n}+2 b\right]-1\right]$. Therefore, we have $\alpha+\beta=-2 n$.

Now using the above relation, (3.9) implies

$$
r=2 n(\alpha-1) .
$$

In [7], Dileo and Pastore proved that in an almost Kenmotsu manifold with $\xi$ belonging to the $(k, \mu)$-nullity distribution the sectional curvature $K(X, \xi)=-1$. From this we get in an almost Kenmotsu manifold with $\xi$ belonging to the $(k, \mu)$ nullity distribution the scalar curvature $r=-2 n(2 n+1)$. Using this value of $r$ we obtain from (3.10), $\alpha=-2 n$. This implies $\beta=0$.

Hence (3.9) reduces to

$$
S(X, W)=-2 n g(X, W) .
$$

From (3.7) we obtain

$$
\begin{aligned}
a R(X, Y) Z= & -b[S(Y, Z) X-S(X, Z) Y+g(Y, Z) Q X-g(X, Z) Q Y] \\
& +\frac{r}{2 n+1}\left[\frac{a}{2 n}+2 b\right][g(Y, Z) X-g(X, Z) Y] .
\end{aligned}
$$

Using the value of $r$ and (3.11) in (3.12) yields

$$
R(X, Y) Z=-[g(Y, Z) X-g(X, Z) Y]
$$

which implies that the manifold is locally isometric to the hyperbolic space $\mathbb{H}^{2 n+1}(-1)$. Conversely, suppose that the manifold is locally isometric to the hyperbolic space 
$\mathbb{H}^{2 n+1}(-1)$. That is, (3.13) holds.

Contracting $X$ in (3.13) yields

$$
S(Y, Z)=-2 n g(Y, Z) .
$$

Hence (3.13) and (3.14) together implies $\tilde{C}(X, Y) Z=0$. That is, the manifold is quasi-conformally flat.

Hence the theorem is proved.

Now, if $a=1$ and $b=-\frac{1}{2 n-1}$, then the quasi-conformal curvature tensor reduces to conformal curvature tensor. Hence we can state the following:

Corollary 3.1. An almost Kenmotsu manifold with $\xi$ belonging to the $(k, \mu)$ nullity distribution is conformally flat if and only if the manifold is locally isometric to the hyperbolic space $\mathbb{H}^{2 n+1}(-1)$.

The above corollary has been proved by De and Mandal [5].

Theorem 3.2. An almost Kenmotsu manifold with $\xi$ belonging to the $(k, \mu)$ - nullity distribution is $\xi$-quasi-conformally flat if and only if the manifold is an Einstein manifold.

Proof: Let us consider a manifold that is $\xi$-quasi-conformally flat. That is,

$$
\tilde{C}(X, Y) \xi=0
$$

which implies

$$
\begin{aligned}
a R(X, Y) \xi= & -b[S(Y, \xi) X-S(X, \xi) Y+g(Y, \xi) Q X-g(X, \xi) Q Y] \\
& +\frac{r}{2 n+1}\left[\frac{a}{2 n}+2 b\right][g(Y, \xi) X-g(X, \xi) Y] .
\end{aligned}
$$

Using (3.2) and (3.4) and $r=-2 n(2 n+1)$ we get from the above equation

$$
\eta(Y) Q X-\eta(X) Q Y=-2 n[\eta(Y) X-\eta(X) Y],
$$

Putting $Y=\xi$ in the above equation we obtain

$$
Q X=-2 n X,
$$

which implies $S(X, Y)=-2 n g(X, Y)$. That is, the manifold is Einstein.

Conversely, assume that the manifold is Einstein. Then there exists a scalar $\lambda$ such that

$$
S(X, Y)=\lambda g(X, Y)
$$

In an almost Kenmotsu manifold with $(k, \mu)$-nullity distribution, the scalar curvature $r=-2 n(2 n+1)$. This implies $\lambda=-2 n$. Now

$\tilde{C}(X, Y) Z=a R(X, Y) Z+b[S(Y, Z) X-S(X, Z) Y+g(Y, Z) Q X-g(X, Z) Q Y]$

$$
-\frac{r}{2 n+1}\left[\frac{a}{2 n}+2 b\right][g(Y, Z) X-g(X, Z) Y] \text {. }
$$


Using (3.18) we get

$$
\tilde{C}(X, Y) Z=a[R(X, Y) Z+(g(Y, Z) X-g(X, Z) Y)] .
$$

Putting $Z=\xi$ in the above equation and using (3.2) we obtain

$$
\tilde{C}(X, Y) \xi=0,
$$

which implies that the manifold is $\xi$-quasi-conformally flat.

If $a=1$ and $b=-\frac{1}{2 n-1}$, then the quasi-conformal curvature tensor reduces to conformal curvature tensor.

Thus we are in a position to state the following:

Corollary 3.2. An almost Kenmotsu manifold with $(k, \mu)$-nullity distribution is $\xi$-conformally flat if and only if it is Einstein.

\section{Quasi-conformally flat almost Kenmotsu manifolds with $\xi$ belonging to the $(k, \mu)^{\prime}$-nullity distribution}

In this section we study $\xi$-quasi-conformally flat almost Kenmotsu manifolds with $\xi$ belonging to the $(k, \mu)^{\prime}$-nullity distribution. Let $X \in \mathcal{D}$ be the eigen vector of $h^{\prime}$ corresponding to the eigen value $\lambda$. Then from $(2.5)$ it is clear that $\lambda^{2}=-(k+1)$, a constant. Therefore $k \leq-1$ and $\lambda= \pm \sqrt{-k-1}$. We denote by $[\lambda]^{\prime}$ and $[-\lambda]^{\prime}$ the corresponding eigenspaces related to the non-zero eigen value $\lambda$ and $-\lambda$ of $h^{\prime}$, respectively. Before presenting our main theorem we recall some results:

Lemma 4.1. (Prop. 4.1 and Prop. 4.3 of [7]) Let $\left(M^{2 n+1}, \phi, \xi, \eta, g\right)$ be an almost Kenmotsu manifold such that $\xi$ belongs to the $(k, \mu)^{\prime}$-nullity distribution and $h^{\prime} \neq 0$. Then $k<-1, \mu=-2$ and Spec $\left(h^{\prime}\right)=\{0, \lambda,-\lambda\}$, with 0 as a simple eigen value and $\lambda=\sqrt{-k-1}$. The distributions $[\xi] \oplus[\lambda]^{\prime}$ and $[\xi] \oplus[-\lambda]^{\prime}$ are integrable with totally geodesic leaves. The distributions $[\lambda]^{\prime}$ and $[-\lambda]^{\prime}$ are integrable with totally umbilical leaves. Furthermore, the sectional curvatures are given by the following:

(a) $K(X, \xi)=k-2 \lambda$ if $X \in[\lambda]^{\prime}$ and $K(X, \xi)=k+2 \lambda$ if $X \in[-\lambda]^{\prime}$,

(b) $K(X, Y)=k-2 \lambda$ if $X, Y \in[\lambda]^{\prime}$; $K(X, Y)=k+2 \lambda$ if $X, Y \in[-\lambda]^{\prime}$ and $K(X, Y)=-(k+2)$ if $X \in[\lambda]^{\prime}, Y \in[-\lambda]^{\prime}$,

(c) $M^{2 n+1}$ has a constant negative scalar curvature $r=2 n(k-2 n)$.

Lemma 4.2. (Lemma 3 of [16]) Let $\left(M^{2 n+1}, \phi, \xi, \eta, g\right)$ be an almost Kenmotsu manifold with $\xi$ belonging to the $(k, \mu)^{\prime}$-nullity distribution. If $h^{\prime} \neq 0$, then the Ricci operator $Q$ of $M^{2 n+1}$ is given by

$$
Q=-2 n i d+2 n(k+1) \eta \otimes \xi-2 n h^{\prime} .
$$

Moreover, the scalar curvature of $M^{2 n+1}$ is $2 n(k-2 n)$. 
From (1.7) we have,

$$
R(X, Y) \xi=k[\eta(Y) X-\eta(X) Y]+\mu\left[\eta(Y) h^{\prime} X-\eta(X) h^{\prime} Y\right],
$$

where $k, \mu \in \mathbb{R}$. Also we get from (4.2)

$$
R(\xi, X) Y=k[g(X, Y) \xi-\eta(Y) X]+\mu\left[g\left(h^{\prime} X, Y\right) \xi-\eta(Y) h^{\prime} X\right] .
$$

Contracting $X$ in (4.2), we have

$$
S(Y, \xi)=2 n k \eta(Y)
$$

Moreover, in an almost Kenmotsu manifold with $(k, \mu)^{\prime}$-nullity distribution

$$
\nabla_{X} \xi=X-\eta(X) \xi+h^{\prime} X
$$

and

$$
\left(\nabla_{X} \eta\right) Y=g(X, Y)-\eta(X) \eta(Y)+g\left(h^{\prime} X, Y\right)
$$

holds.

Theorem 4.1. $A(2 n+1)$-dimensional $(n>1)$ quasi-conformally flat almost Kenmotsu manifold with $\xi$ belonging to the $(k, \mu)^{\prime}$-nullity distribution is either conformally flat or of a quasi-constant curvature.

Proof: Let us assume that the manifold $M^{2 n+1}$ is quasi-conformally flat, that is,

$$
\tilde{C}(X, Y) Z=0
$$

for any vector fields $X, Y, Z$ on $M^{2 n+1}$.

From (1.3) we have

$$
\begin{aligned}
a \tilde{R}(X, Y, Z, W)= & b[S(X, Z) g(Y, W)-S(Y, Z) g(X, W) \\
& +S(Y, W) g(X, Z)-S(X, W) g(Y, Z)] \\
& +\frac{r}{(2 n+1)}\left[\frac{a}{2 n}+2 b\right][g(Y, Z) g(X, W)-g(X, Z) g(Y, W)] .
\end{aligned}
$$

Putting $Z=\xi$ in the above equation and using (4.2) and (4.4) we have

$$
\begin{array}{r}
a k[\eta(Y) g(X, W)-\eta(X) g(Y, W)]+a \mu\left[\eta(Y) g\left(h^{\prime} X, W\right)-\eta(X) g\left(h^{\prime} Y, W\right)\right] \\
=b[2 n k \eta(X) g(Y, W)-2 n k \eta(Y) g(X, W)-\eta(Y) S(X, W)+\eta(X) S(Y, W)] \\
+\frac{r}{(2 n+1)}\left[\frac{a}{2 n}+2 b\right][\eta(Y) g(X, W)-\eta(X) g(Y, W)] .
\end{array}
$$


Putting $Y=\xi$ in the above equation and using (4.4) we get after simplifying

$$
\begin{aligned}
S(X, W)= & {\left[-2 n k+\frac{r}{b(2 n+1)}\left[\frac{a}{2 n}+2 b\right]-\frac{a k}{b}\right] g(X, W) } \\
& +\left[4 n k-\frac{r}{b(2 n+1)}\left[\frac{a}{2 n}+2 b\right]+\frac{a k}{b}\right] \eta(X) \eta(W)-\frac{a \mu}{b} g\left(h^{\prime} X, W\right) .
\end{aligned}
$$

Let us denote

$$
A=-2 n k+\frac{r}{b(2 n+1)}\left[\frac{a}{2 n}+2 b\right]-\frac{a k}{b}
$$

and

$$
B=4 n k-\frac{r}{b(2 n+1)}\left[\frac{a}{2 n}+2 b\right]+\frac{a k}{b} .
$$

Then, we see that

$$
A+B=2 n k .
$$

Putting $X=W=e_{i}$ in (4.10), where $\left\{e_{i}\right\}$ is an orthonormal basis of the tangent space at each point of the manifold and taking summation over $i, i=1,2,3 \ldots .,(2 n+$ 1), we get

$$
r=A(2 n+1)+B .
$$

From (4.13) and (4.14) we get

$$
A=\frac{r}{2 n}-k .
$$

From (4.11) and (4.15), it follows that

$$
-2 n k+\frac{r}{b(2 n+1)}\left[\frac{a}{2 n}+2 b\right]-\frac{a k}{b}=\frac{r}{2 n}-k .
$$

The above relation gives

$$
(a+2 n b-b)(r-2 n k(2 n+1))=0 .
$$

Hence, either $a+2 n b-b=0$ or $r=2 n k(2 n+1)$.

Let us suppose that $a+2 n b-b=0$. Then we see that $b=-\frac{a}{2 n-1}$. Hence, from (1.3), it follows that $\tilde{C}(X, Y) Z=a C(X, Y) Z$, where $C(X, Y) Z$ is the Weyl conformal curvature tensor. So, in this case, the quasi-conformally flat manifold is conformally flat. 
Now, if $r=2 n k(2 n+1)$, then from (4.10) we obtain

$$
S(X, W)=2 n k g(X, W)-\frac{a \mu}{b} g\left(h^{\prime} X, W\right) .
$$

Using (4.17) in (4.8) yields

$$
\begin{aligned}
\tilde{R}(X, Y, Z, W)= & k[g(Y, Z) g(X, W)-g(X, Z) g(Y, W)] \\
& -\mu\left[g\left(h^{\prime} X, Z\right) g(Y, W)-g\left(h^{\prime} Y, Z\right) g(X, W)\right. \\
& \left.+g\left(h^{\prime} Y, W\right) g(X, Z)-g\left(h^{\prime} X, W\right) g(Y, Z)\right] .
\end{aligned}
$$

From (4.1) and (4.17), it follows that

$$
g\left(h^{\prime} X, W\right)=l[g(X, W)-\eta(X) \eta(W)],
$$

where $l=\frac{2 n b(k+1)}{a \mu-2 n b}=-\frac{n b(k+1)}{a+n b}$, by Lemma 4.1 .

Using (4.19) in (4.18) we get

$$
\begin{aligned}
\tilde{R}(X, Y, Z, W)= & p[g(Y, Z) g(X, W)-g(X, Z) g(Y, W)] \\
& +q[g(X, W) \eta(Y) \eta(Z)-g(X, Z) \eta(Y) \eta(W) \\
& +g(Y, Z) \eta(X) \eta(W)-g(Y, W) \eta(X) \eta(Z)],
\end{aligned}
$$

where $p=k-4 l$ and $q=2 l$.

This completes the proof.

\section{Extended quasi-conformal curvature tensor of an almost Kenmotsu manifold with $(k, \mu)$-nullity distribution}

In this section we study vanishing extended quasi-conformal curvature tensor and extended $\xi$-quasi-conformally flat almost Kenmotsu manifolds with $\xi$ belonging to $(k, \mu)$-nullity distribution.

The extended form of quasi-conformal curvature tensor can be written as

$$
\begin{aligned}
\tilde{C}_{e}(X, Y) Z= & a R(X, Y) Z+b[S(Y, Z) X-S(X, Z) Y+g(Y, Z) Q X-g(X, Z) Q Y] \\
& -\frac{r}{2 n+1}\left[\frac{a}{2 n}+2 b\right][g(Y, Z) X-g(X, Z) Y] \\
& -\eta(X) \tilde{C}(\xi, Y) Z-\eta(Y) \tilde{C}(X, \xi) Z-\eta(Z) \tilde{C}(X, Y) \xi .
\end{aligned}
$$

Theorem 5.1. In an almost Kenmotsu manifold with $\xi$ belonging to $(k, \mu)$-nullity distribution, the extended quasi-conformal curvature tensor vanishes if and only if the manifold is locally isometric to the hyperbolic space $\mathbb{H}^{2 n+1}(-1)$.

Proof: Putting $Y=Z=\xi$ and supposing that the extended quasi-conformal tensor vanishes, we get from (5.1)

$$
\begin{array}{r}
a R(X, \xi) \xi+b[S(\xi, \xi) X-S(X, \xi) \xi+Q X-\eta(X) Q \xi]+(a+4 n b)(X-\eta(X) \xi) \\
(5.2) \quad-\eta(X) \tilde{C}(\xi, \xi) \xi-\tilde{C}(X, \xi) \xi-\tilde{C}(X, \xi) \xi=0 .
\end{array}
$$


Now, using (3.4) and (3.5) the above equation reduces to

$$
b Q X=-2 n b X+2 \tilde{C}(X, \xi) \xi .
$$

Now, Using (3.2), (3.4) and (3.5) we obtain

$$
\tilde{C}(X, \xi) \xi=2 n b X+b Q X .
$$

Putting the value of $\tilde{C}(X, \xi) \xi$ in (5.3) we get

$$
Q X=-2 n X,
$$

which implies

$$
S(X, Y)=-2 n g(X, Y) .
$$

This shows that the manifold is Einstein. Since, the extended quasi-conformal curvature tensor vanishes, we have from (5.1)

$$
\begin{aligned}
a R(X, Y) Z= & -b[S(Y, Z) X-S(X, Z) Y+g(Y, Z) Q X-g(X, Z) Q Y] \\
& -(a+4 n b)[g(Y, Z) X-g(X, Z) Y] \\
& +\eta(X) \tilde{C}(\xi, Y) Z+\eta(Y) \tilde{C}(X, \xi) Z+\eta(Z) \tilde{C}(X, Y) \xi .
\end{aligned}
$$

Now, making use of (3.3), (3.4), (3.5) and (5.5) we obtain

$$
\tilde{C}(\xi, Y) Z=0, \tilde{C}(X, \xi) Z=0 .
$$

Again since the manifold is Einstein, we have from Theorem 3.2

$$
\tilde{C}(X, Y) \xi=0 .
$$

Putting these values in (5.7) and using (5.6) we get

$$
R(X, Y) Z=-[g(Y, Z) X-g(X, Z) Y] .
$$

This implies that the manifold is locally isometric to the hyperbolic space $\mathbb{H}^{2 n+1}(-1)$.

Conversely, suppose that the manifold is locally isometric to the hyperbolic space $\mathbb{H}^{2 n+1}(-1)$. That is, (5.8) holds.

Contracting $X$ in (5.8) yields

$$
S(Y, Z)=-2 n g(Y, Z) .
$$

Now, as shown earlier in this theorem

$$
\tilde{C}(\xi, Y) Z=\tilde{C}(X, \xi) Z=\tilde{C}(X, Y) \xi=0 .
$$

Then, making use of (5.8), (5.9) and the above values, we obtain from (5.1) that

$$
\tilde{C}_{e}(X, Y) Z=0 .
$$

Hence the theorem is proved. 
Theorem 5.2. An almost Kenmotsu manifold with $\xi$ belonging to the $(k, \mu)$-nullity distribution is extended $\xi$-quasi-conformally flat if and only if the manifold is Einstein.

Proof: Suppose $\tilde{C}_{e}(X, Y) \xi=0$ and putting $Y=\xi$, we get from (5.1)

$$
\begin{array}{r}
a R(X, \xi) \xi+b[S(\xi, \xi) X-S(X, \xi) \xi+Q X-\eta(X) Q \xi]+(a+4 n b)(X-\eta(X) \xi) \\
(5.10) \quad-\eta(X) \tilde{C}(\xi, \xi) \xi-\tilde{C}(X, \xi) \xi-\tilde{C}(X, \xi) \xi=0 .
\end{array}
$$

Now, using (3.4) and (3.5) the above equation reduces to

$$
b Q X=-2 n b X+2 \tilde{C}(X, \xi) \xi .
$$

Now, Using (3.2), (3.4) and (3.5) we obtain

$$
\tilde{C}(X, \xi) \xi=2 n b X+b Q X
$$

Putting the value of $\tilde{C}(X, \xi) \xi$ in $(5.11)$ we get

$$
Q X=-2 n X
$$

which implies that the manifold is Einstein.

Conversely, if the manifold is Einstein then obviously $\tilde{C}_{e}(X, Y) \xi=0$.

Hence the theorem is established.

Acknowledgement: The authors are thankful to the referees for their valuable suggestions towards the improvement of this paper. The author Dibakar Dey is supported in part by CSIR, India (File no: 09/028(1010)/2017-EMR-1).

\section{R E F E R E N C E S}

1. D. E. Blair, T. Koufogiorgos and B. J. Papantoniou: Contact metric manifolds satisfying a nulitty condition, Israel. J. Math. 91(1995), 189-214.

2. D. E. BLAIR: Contact manifold in Reimannian geometry, Lecture notes in Mathematics, Springer, Berlin, 509(1976).

3. D. E. BLAIR: Reimannian geometry of contact and symplectic manifolds, Birkhäuser, Boston, 2002.

4. B. Y. Chen and K. YAno: Hypersurfaces of a conformally flat space, Tensor (N.S) 26(1972), 318-322.

5. U. C. DE and K. MANDAL: On a type of almost Kenmotsu manifolds with nullity distributions, Arab J. Math. Sci. 23(2017), 109-123.

6. U. C. De and K. MANDAL: On $\phi$-Ricci recurrent almost Kenmotsu manifolds with nullity distributions, Int. Electron. J. Geom. 9(2016), 70-79.

7. G. Dileo and A. M. PAstore: Almost Kenmotsu manifolds and nullity distributions, J. Geom. 93(2009), 46-61. 
8. G. Dileo and A. M. PAstore: Almost Kenmotsu manifolds with a condition of $\eta$-parallelsim, Differential Geom. Appl. 27(2009), 671-679.

9. G. Dileo and A. M. PAstore: Almost Kenmotsu manifolds and local symmetry, Bull. Belg. Math. Soc. Simon Stevin 14(2007), 343-354.

10. A. Gray: Spaces of constancy of curvature operators, Proc. Amer. Math. Soc. 17(1966), 897-902.

11. K. Kenmotsu: A class of almost contact Riemannian manifolds, Tohoku Math. J., 24(1972), 93-103.

12. K. MANDAL and U. C. DE: On some classes of 3-dimensional normal almost paracontact metric manifolds, Southeast Asian Bull. Math. 41(2017), 231-238.

13. A. M. Pastore and V. Saltarelli: Generalized nullity distribution on almost Kenmotsu manifolds, J. Geom. 4(2011), 168-183.

14. A. M. Pastore and V. Saltarelli: Almost Kenmotsu manifolds with conformal Reeb foliation, Bull. Belg. Math. Soc. Simon Stevin 21(2012), 343-354.

15. S. TAnno: Some differential equations on Riemannian manifolds, J. Math. Soc. Japan, 30(1978), 509-531.

16. Y. WANG and X. LIU: on $\phi$-recurrent almost Kenmotsu manifolds, Kuwait J. Sci. 42(2015), 65-77.

17. Y. WANG and X. LIU: Second order parrallel tensors on almost Kenmotsu manifolds satisfying the nullity distributions, Filomat 28(2014), 839-847.

18. Y. WANG and X. LIU: Riemannian semi-symmetric almost Kenmotsu manifolds and nullity distributions, Ann. Polon. Math. 112(2014), 37-46.

19. Y. WAng: Conformally flat almost Kenmotsu 3-manifolds, Med. J. Math. 14(2017):186.

20. Y. WANG: Three dimensional almost Kenmotsu manifolds with $\eta$-parallel Ricci tensors, J. Korean Math. Soc. 54(2017), 793-805.

21. K. YANO and S. SAWAKI: Riemannian manifolds admitting a conformal transformation group, J. Differential Geom. 2(1968), 161-184.

22. G. Zhen, J. L. Cabrerizo, L. M. FernÁndez and M. FernÁndez: On $\xi$ conformally flat contact metric manifolds, Indian J. Pure Appl. Math. 28(1997), $725-734$

Dibakar Dey

Department of Pure Mathematics

University of Calcutta

35, Ballygunge Circular Road, Kol- 700019

deydibakar3@gmail.com

Pradip Majhi

Department of Pure Mathematics

University of Calcutta

35, Ballygunge Circular Road, Kol- 700019

mpradipmajhi@gmail.com 